\title{
O arabskiej literaturze podróżniczej
}

\author{
Z Profesor Roswithą Badry* \\ rozmawia Magdalena Lewicka
}

DOI: http://dx.doi.org/1 0.12775/LC.2019.015

Magdalena Lewicka: Frau Professor, das Thema des vorliegenden Bandes ist eines der ältesten Motive in der Literatur, das Schriftsteller aller Epochen und unterschiedlicher Breitengrade in ihrem Schaffen benutzt haben. Besprochen und diskutiert werden hier literarische Verarbeitungsformen des Phänomens Reisen im Schrifttum des Okzidents und Orients, wobei der Terminus Orient auf die arabische bzw. arabisch-muslimische Welt beschränkt ist. Die Betrachtungen der Autoren der hier versammelten, in einer literaturwissenschaftlichen Perspektive eingebetteten Texte knüpfen an den Topos der Reise an - sowohl in ihrer wirklichen Dimension, d.h. einer Wanderung, die aus welchen Beweggründen auch immer vorgenommen wird, zu diesem oder irgendeinem anderen Zweck, entweder freiwillig oder erzwungen durch Emigration und Exil, mit all ihren Implikationen - als auch in ihrer symbolischen, metaphysischen und metaphorischen Dimension, d.h. einer Reise in Zeit oder Raum, die zur Reflexion über sich selbst und über das eigene Schicksal führt, auf der Suche nach Werten des gegenwertigen Seins und nach dem Sinn der menschlichen Existenz, bzw. als Flucht vor den Problemen des Lebens, persönlichen Dilemmas und/oder dem inneren Zerrissen-Sein. Im Gespräch mit Ihnen, Frau Professor, möchte ich jedoch an die erstgenannte Auffassung von Reise anknüpfen, das heißt einer wirklichen Reise und der dabei entstehenden Reflexion, und zwar im Kontext der arabischen Reiseliteratur, die eine vorzügliche und zugleich überaus interessante Widerspiegelung dessen bildet, und - wie es scheint - dem polnischen bzw. deutschen Leser immer noch wenig bekannt ist. Dieses Schrifttum ist eine der Strömungen, der sich seit dem 9. Jh. entwickelnden arabischen geographischen Literatur ihre Entfaltung wurde durch die große Mobilität der mittelalterlichen Araber und anderer

\footnotetext{
* Dr hab. nauk humanistycznych w zakresie orientalistyki, islamoznawstwa, profesor Albert-LudwigsUniwersität Freiburg. E-mail: roswitha.badry@orient.uni-freiburg.de.
} 
Bewohner des Kalifenreiches gefördert, die auf dem damals weiten Gebiet des islamisch geprägten Territoriums und außerhalb dessen hin und her wanderten. Welche Faktoren lagen der Aufnahme von solchen Reisen zugrunde?

Roswitha Badry (nachfolgend: R.B.): Als eines der herausragenden (persönlichen) Motive für die Aufnahme weiter, beschwerlicher Reisen ist wohl die „Suche nach Wissen” (țalab al-'ilm) zu nennen. Wie eine oft zitierte, angeblich prophetische Überlieferung besagt, sollte der Muslim (überall) nach Wissen suchen, „selbst wenn es in China sei”. Und tatsächlich haben viele Wissenssucher sich auf lange Reisen begeben, um bei einem bestimmten, renommierten Lehrer zu hören. Zudem stellte die Pilgerfahrt zu den heiligen Stätten des Islam - an erster Stelle natürlich Mekka und Medina, nicht zu vergessen aber Jerusalem oder schiitische Pilgerorte, vornehmlich im Irak oder in Iran - einen wichtigen Auslöser für Reisen dar. Zuweilen mögen auch beide (religiös bedingte) Anliegen den Anlass für das Reisen gebildet haben, manchmal in Kombination mit Handels- oder auch Missionierungsinteressen (wie z.B. bei den fatimitidischen „Werbern”, die sich oft als Händler tarnten). So manch einen hat Wissbegierde, Neugierde sowie Abenteuerlust zu weitergehenden Reisen veranlasst, ebenfalls oft verbunden mit Handels-, Lehr- oder richterlichen Tätigkeiten, allein schon um das Weiterreisen zu finanzieren. Einen anderen Auslöser haben Sie bereits selbst angesprochen: die erzwungene Reise - d.h. um einer drohenden Strafe zu entgehen oder um politischer Unterdrückung, wirtschaftlichen oder ähnlichen Krisen zu entfliehen. Nicht zu vergessen ist der ursprüngliche (öffentliche) Anreiz pragmatischer, geopolitischer Natur: Gemeint sind die Verwaltungs- und Handelsbedürfnisse des arabisch-islamischen Großreichs, darunter die Notwendigkeit der Schätzung des Steuereinkommens einzelner Regionen, die Gewährung bzw. Überprüfung der Sicherheit der Wegenetze, sowohl für die Pilger- und Handelskarawanen als auch für nachrichtendienstliche Tätigkeiten oder die Einrichtung von Poststationen.

Ein Impuls zur Entstehung der arabischen erdkundlichen Literatur waren Übersetzungen der indischen und persischen Werke, erwähnt wird dabei auch der Einfluss des Gedankengutes der griechischen Literatur. Welche Autoren haben die Araber am frühesten kennengelernt? Welche Werke lagen der Herausbildung des geographischen Schrifttums zugrunde?

R.B.: Zum einen ist das antike griechische Erbe zu nennen, das für die Entwicklung der mathematischen und physischen Geographie (Geodäsie, Kartographie) maßgeblich war, wie vielleicht bereits das arabische Lehnwort für Geographie andeutet ( $\check{g} u \bar{g} r a \bar{a} f i y \bar{a})$. Neben der Geographie des Marinos von Tyros ist dabei besonders das Werk des Claudius Ptolemäus zu erwähnen. Zum anderen wurden Erkenntnisse und Anregungen aus der geographischen Literatur Persiens und Indiens einbezogen, wobei indische Vorbilder zwar nachweisbar sind, nicht aber im selben Maße wie in der Astronomie. Diese Übernahmen oder Adaptionen waren eine Folge der Übersetzertätigkeiten, die unter dem abbasidischen Kalifen Al-Ma'mūn (813-833) und dem von ihm errichteten „Haus der Weisheit” (Bayt al-Hikma) eine erste Hochblüte erleben sollten. Später kamen z. B. Übersetzungen im muslimischen Spanien (Al-Andalus) hinzu. In diesem Zusammenhang scheint es angebracht hervorzuheben, was für eine große Offenheit arabische bzw. muslimische Gelehrte außerislamischem Gedankengut entgegengebracht haben. Ähnliches gilt für die gleichzeitig erstellten eigenen Forschungsarbeiten im Rahmen der deskriptiven Geographie, zu der 
Historiker und Verwaltungsbeamte ebenso beigetragen haben wie Kaufleute, Seefahrer oder religiöse Gelehrte. Diese Beschreibungen sind in der Regel durch eine überraschend neutrale Sachlichkeit gekennzeichnet.

Die Werke des arabischen erdkundlichen Schrifttums werden gewöhnlich in Anlehnung an zwei Schulen charakterisiert: die irakische Schule und die Schule von Al-Balhīi. Wo verläuft die Trennlinie zwischen beiden Schulen, und worauf beruht diese Unterscheidung?

R.B.: Grob gesagt wird der Unterschied im Allgemeinen an Inhalt, Methode und Blickwinkel der beiden Schulen festgemacht. Während der Balhī-Schule eine eher religiös-islamisch ausgerichtete Orientierung zugesprochen wird, die Mekka und Medina ins Zentrum rückt, wird der irakischen eine weitgehend 'säkulare' Ausrichtung nachgesagt, welche den Irak und besonders Bagdad in den Fokus ihrer Betrachtung stellt und stärker von vorislamischen, persisch-sassanidischen Vorbildern geprägt ist. Ob man diese Differenzierung generalisieren oder überbetonen sollte, sei einmal dahingestellt.

Welches Werk wird als das früheste Werk der arabischen geographischen Literatur erwähnt, und was ist sein Inhalt?

R.B.: Als das früheste Werk der geographischen Literatur gilt das „Buch der Gestalt der Erde" (Kitāb șūrat al-'arḍ) des Mathematikers, Astronomen und Geographen Abū Ğa'far al-Huwārizmī (gest. um 850), auch bekannt unter seinem latinisierten Namen Algorismi, das er 833 beendete. Wie sein Name andeutet, stammte er ursprünglichen aus dem damaligen Osten Irans, was ebenfalls auf die anfängliche Dominanz von Autoren mit iranischem Hintergrund, geprägt von der persischen Administrations- und Bildungstradition,

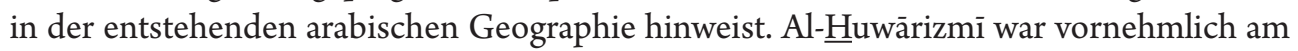
oben genannten Haus der Weisheit in Bagdad tätig. Bei seinem geographischen Hauptwerk handelt es sich um eine systematisierte und bearbeitete Fassung der Geographie des Ptolemäus. Abgesehen von der Korrektur der geographischen Vorstellungen des Ptolemäus (so z.B. von dessen Angaben zum Mittelmeer) unterteilte er - im Anschluss an das sassanidische System der sieben „Regionen” - die damals bekannte bewohnte Erde in sieben Zonen („Klimata“ bzw. Längszonen von West nach Ost). Diese Einteilung sollte späteren Geographen als Vorbild dienen.

Wem werden die ersten Beschreibungen von Ländern, Wegen und Königreichen zugeschrieben, die mit dem Terminus kitāb al-masālik wa-al-mamälik oder kitāb al-buldān bezeichnet werden? Welchen Charakter tragen diese Werke und welche Regionen beschreiben sie? Bis zu welchem Grad sind sie in der beschreibenden Erdkunde begründet? Und inwieweit bewegen sie sich an der Grenze zwischen geographischem Schrifttum und didaktischer Adab-Literatur, in der Prosa mit Poesie, Ernst mit Humor verflochten sind, während inhaltlich betrachtet in Anekdoten, Erzählungen, Berichten und Gedichten verschiedene Phänomene, Menschen und Menschengruppen, Ortschaften und Ereignisse präsentiert werden. Und all das dient der Bildung und Unterhaltung?

R.B.: Ibn Hurradādbihs Buch der Reiserouten und der Reiche gilt als das älteste erhaltene Werk der administrativen Geographie. Auch dieser Autor stammte ursprünglich 
aus Iran, wuchs aber in Bagdad auf und war in mehreren Provinzen für den Post- und Nachrichtendienst des abbasidischen Kalifen im 9. Jh. tätig. Die in dieser Position gesammelten Erfahrungen kamen ihm bei der Abfassung des genannten Werkes zugute, dessen erste Version Mitte des 9. Jahrhunderts vorlag. Darin gliederte er die Erde in vier Teile, grob in einen nördlichen und einen südlichen, die er wiederum in sieben Klimata unterteilte. Beginnend mit dem Irak verwies er auf östliche, westliche, weiter nördlich liegende Regionen sowie auf die südlich gelegene Region der Arabischen Halbinsel. Im Mittelpunkt standen für ihn die Wegstationen und die Entfernungen zwischen ihnen; bei Regionen im

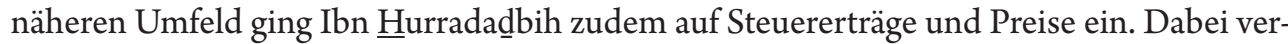
gas er nicht, allerlei Verse, legendäres, sagenhaftes und anekdotisches Material einzuflechten - ganz im Stil des Gebildeten, des Adīb, bzw. Literaten. Diese Verbindung von Fakt und Fiktion, Akademischem bzw. Wissenschaftlichem oder Nützlichem mit Unterhaltsamen ist typisch für einen Großteil der geographischen, aber auch historiographischen Literatur, die ja beide eng miteinander verwoben sind, deren Autoren auch oft dieselben sind. Ganz anders verhält es sich in der Regel mit den als Buch der Länder betitelten Werken. Dazu zählt unter anderem das nur in Teilen auf uns gekommene Werk von Al-Ya'qūbì (gest. 897), der als Historiker und höfischer Sekretär stärker auf historische Informationen als auf geographische fokussiert war und ausschmückende Passagen unterließ. Demgegenüber ist das gleichnamige Werk des Ibn al-Faqīh al-Hamad̄ānī, ca. 903 verfasst, stärker an den unterhaltsamen Komponenten interessiert. Sein Buch steht damit eher in der Adab-Tradition als in der wissenschaftlicher Abhandlungen zur Geographie.

Als das wichtigste Werk der beschreibenden arabischen Erdkunde wird das Kitāb ahssan at-taqāsīm fī ma'rifat al-aqālìm von Al-Muqaddasī angesehen, welches der Autor mit Lob des eigenen Werkes abgeschlossen hat, indem er meinte, dass es sich um „ein Buch handelt, das wie Perlen und Frühjahr, wie Gemmen und schöne Gärten den Menschen erfreut”. Was begegnet dem Leser, wenn er sich auf den Spaziergang durch jene „schönen Gärten” begibt? Und wie sind jene ihn „erfreuenden Perlen"?

R.B.: Der von Ihnen zitierte Schlusssatz des genannten Werks ist als eine Art Werbung in eigener Sache zu verstehen. Der Autor aus dem 10. Jh. war ein weitgereister Kaufmann, dem Namen nach aus Jerusalem, und Sohn eines Baumeisters der Fatimiden. Al-Muqaddasī knüpfte zwar an die Schriften seiner Vorgänger an, war aber von dem Ehrgeiz beseelt, aus der Geographie eine „nützliche Wissenschaft“ zu machen, die sich gleichermaßen an Händler wie die Bildungselite wendet. Insgesamt strebte der Verfasser eine gröBere Systematik in Theorie und Methode an und wollte sich von bisheriger Landeskunde und Kosmographie absetzen. Er stellte die arabische Geographie auf eine neue wissenschaftliche Basis auf der Grundlage empirischer Beobachtungen und einer eigenen geographischen Terminologie. In seinem Werk deckte er bereits alle Felder der heutigen Geographie ab: die physische und besonders die Human- und Anthropogeographie mit den Unterdisziplinen politische, Sozial- und Wirtschafts- sowie Kulturgeographie. Sein Buch bietet $\mathrm{u}$. a. wertvolle Informationen zu anthropogeographischen Sachverhalten wie Sprache, Religion, Lebensverhältnisse. Im Mittelpunkt steht die islamische Welt, die er in sechs arabische Provinzen bzw. Klimata und acht nichtarabische einteilte. Der Autor beschreibt eine jede Provinz und ihre Städte mit Angaben zum Klima, zu den Produkten, 
Gewässern, Bergwerken, Gebäuden, Heiligen Stätten, den Münzen, Steuern, Gewichten, Sitten und Bräuchen der Bewohner, politischen Machtverhältnissen, Schulen, Wegen und Routen. Bagdad rühmt er als Einheitssymbol über die Maße in kunstvoller Reimprosa, allerdings „aus Konvention“, wie er selbst eingesteht, denn schließlich bemerkt er, dass der Ruhm von Bagdad mittlerweile verblasst und heute vielmehr auf das von den Fatimiden begründete Kairo (Al-Fusțāț) bzw. Cordoba übergegangen sei.

Eine besondere Bedeutung wird auch dem Buch $M u^{\prime}$ '̆am al-buldān von Yãqūt alHamawī zugeschrieben, das als Kompendium der geschichtlichen Erdkunde angesehen wird, zugleich als eine überaus interessante Quelle mit kultur-gesellschaftlichem Charakter. Interessant ist die Begründung, mit welcher der Autor die Faktoren nennt, die ihn zum Schreiben dieses Werkes bewegt haben. Könnten Sie, Frau Professor, den Inhalt dieses Kompendiums uns näher erläutern und charakterisieren?

R.B.: In seinem Lexikon listet Yāqūt (gest. 1229 in Aleppo) in alphabetischer Reihenfolge die aus verschiedenen Werken der arabischen Literatur bekannten Orte und Regionen auf, weil er, wie er einleitend bemerkt, den koranischen Passagen zu Gottes Schöpfung und der Aufforderung an die Muslime zu reisen nachkommen wolle, und nicht in erster Linie der Unterhaltung dienen möchte. In dieser Einführung finden sich zudem Begriffserklärungen sowie Ausführungen zur Kosmographie. Selbst wenn sein Hauptansinnen nicht das der Unterhaltung ist - von einem Nachschlagewerk ist das auch nicht zu erwarten - so hat Yāqūt dennoch Anekdoten zu den Regionen des Kalifats und seinen Bewohnern eingestreut. Er selbst hatte auf seinen zahlreichen Reisen tiefe Einblicke gewonnen, kritisierte und korrigierte fehlerhafte bzw. ungeprüft übernommene Angaben in früheren historischen oder biographischen Werken. Das Lexikon enthält neben Informationen zur Geographie und zu den Ortsnamen (Toponomastik) literarische und poetische Themen sowie biographische Details zu prominenten Persönlichkeiten, die in den beschriebenen Orten gelebt haben. Damit hat der Autor ein bis heute für den Arabisten und Islamwissenschaftler unverzichtbares Nachschlagewerk hinterlassen.

Als erste eigentliche Reisebeschreibung in der arabischen Literatur gilt Kitäb von Ibn Faḍlān, in dem er die in den Jahren 921-922 unternommene Reise gen Norden in das Land der Wolga-Bulgaren dargestellt hat. Was weiß man über den Autor und seinen Bericht?

R.B.: Über den Autor ist so gut wie nichts bekannt, außer dem, was er selbst in seinem Reisebericht vermerkt hat. Demnach handelte es sich um eine Gesandtschaft im Auftrag des Kalifen Al-Muqtadir (reg. 908-933) zum König der Wolga-Bulgaren, der kurz zuvor den Islam angenommen hatte. Dem Autor kam seinen eigenen Ausführungen nach die Aufgabe zu, dem König den Brief des Kalifen vorzutragen, Geschenke an den Herrscher und seine Entourage zu übergeben sowie die Juristen und Lehrer zu kontrollieren, die der Kalif auf Bitte des Königs mitgeschickt hatte, um den Wolga-Bulgaren die Gesetze des Islam zu vermitteln. Die Wegstationen der Hinreise 921-922 werden in dem Bericht ausführlich beschrieben (Buchara - Chwarezm - Gurgandsch), während genaue Daten zur Rückreise fehlen, was auch mit der Unvollständigkeit der den Editionen zugrunde gelegten Handschrift aus Maschhad (Nordostiran), die 1823 entdeckt worden war, zusammenhängen mag. Auf jeden Fall enthält das Werk wertvolle ethnographische Angaben zu den 
heidnischen Turkvölkern (Oghuz), durch deren Regionen die Gesandtschaft kam, zu den warägischen Rus (den aus Skandinavien stammenden Händlern, die unter anderem im Wolga-Raum tätig waren), den Khazaren (den südlichen Nachbarn der Wolga-Bulgaren) und selbstverständlich den Wolga-Bulgaren, deren Sitten, Gebräuche und Lebensumstände genau vom Verfasser beobachtet und beschrieben wurden. Der zuvor erwähnte Yāqūt hat, wie er selbst einräumt, die Ausführungen des Ibn Faḍlān über diese Völker Wort für Wort übernommen.

An dieser Stelle lohnt es sich daran zu erinnern, dass das Werk ins Polnische übersetzt wurde: Ibn Faḍlān, Kitāb, bearbeitet von A. Kmietowicz, F. Kmietowicz, T. Lewicki, Wrocław 1985. In der Einleitung zu der Übersetzung ist zu lesen: „Vom Standpunkt der Forschungen zur Geschichte, Erdkunde und Ethnographie von Osteuropa im 10. Jh. liefert uns der Bericht von Ibn Faḍlān ein Material, dessen Bedeutung nur schwer zu überschätzen ist. Im Gegensatz nämlich zu den meisten zeitgenössischen mittelalterlichen arabischen Quellen, die sich auf Osteuropa beziehen und sich an mittelbare, aus zweiter Hand bekannte Berichte anlehnen, die von Schriftstellern auf dem Gebiet des Kalifats gewonnen wurden, stammte der Bericht [von Ibn Fạ̣lān] aus der Feder eines unmittelbaren Beobachters der zu beschreibenden Situationen und Ereignisse. Sie ist die erste Reportage in der arabischen Literatur über eine Reise auf diese Gebiete, die von einem Augenzeugen niedergeschrieben wurde, und dazu noch mit einer präzisen Datierung und mit einer sehr zusammenhängenden Chronologie”. Wer setzte das Werk von Ibn Fạ̣lāns fort und welche Nachfolger hat er gefunden?

R.B.: Auch aus der Folgezeit sind Reiseberichte von Gesandten in diplomatischer Mission, von Pilgern, Gelehrten, Händlern oder Seefahrern (vgl. nautische Literatur) bezeugt, wenngleich deren systematische wissenschaftliche Aufarbeitung bisher - so zumindest meines Wissens nach - nur in Einzelfällen erfolgt ist.

Als ein Muster für Werke dieses Typs wurde die Riḥla von Ibn Ğubayr anerkannt, dessen Handlungsachse seine Reise nach Mekka in den Jahren 1 183-1 185 ist, die vom Autor durch den Bedarf begründet wurde, eine Bußpilgerfahrt zu absolvieren, dafür dass er sich zum Weintrinken verleiten ließ. Daher ist ein Drittel der Beschreibung den Wallfahrtsriten und dem Leben in den heiligen Städten Mekka und Medina gewidmet, in denen er über ein Jahr verbracht hatte. Erst nach einem abenteuerreichen Aufenthalt in Syrien und Irak sollte er nach Spanien zurückkehren. Welches ist das berühmteste Werk aus der Rihla-Reihe?

R.B.: Als berühmtestes Werk des Rihla-Genres gilt der Bericht des weitgereisten Nordafrikaners, genauer gesagt aus der Hafenstadt Tanger in Nordmarokko stammenden Ibn Bațtuța, den seine insgesamt über 27 Jahre andauernden Reisen im 14. Jh. - etwa ein halbes Jahrhundert nach Marco Polo - unter anderem bis nach Süd- und Südostasien, China und verschiedene afrikanische Länder führten. Keiner war vor ihm so weit gereist. Deshalb wird er auch als „Marco Polo der Araber“ bezeichnet. Nach seiner Rückkehr diktierte er seine Erlebnisse dem Hofsekretär des damaligen Herrschers (des Meriniden-Sultans) von Fez, dem Dichter Ibn Ğuzayy, der das Werk redigierte und edierte, hier und da zudem Ergänzungen einfügte. Auch wenn die Informationen in dem Werk über das Alltagsleben 
in vielen Regionen recht informativ sind, so ist doch fraglich, inwieweit alle Erlebnisse auf eigener Anschauung beruhen. Zuweilen hat Ibn Bațtūṭa seine Erinnerungen eindeutig mit Fantasien, Legenden und Mythen ausgeschmückt; an anderen Stellen ist nicht genau festzustellen, ob er Tatsachen oder Märchen erzählt. Gleichwohl stehen die Schilderungen seiner Fahrten mit Blick auf Entfernung und Zeitdauer sowie in ihrem literarischen Ertrag in nichts dem weltberühmt gewordenen Reisebericht des venezianischen Händlers Marco Polo nach. Dessen Bericht ist ja, was die Glaubwürdigkeit betrifft, ebenfalls teilweise von der Wissenschaft angezweifelt worden.

Sind in den nachfolgenden Jahrhunderten genauso wertvolle Reisebeschreibungen entstanden?

R.B.: Auch aus den nachfolgenden Jahrhunderten sind Reiseberichte belegt, darunter nun auch in persischer oder osmanisch-türkischer Sprache. Dennoch sind die Reiseberichte, die seit dem 19. Jh. verfasst wurden, wohl eher einem breiteren Publikum bekannt, entweder aus Übersetzungen oder auch wegen der mehrfachen Neuauflagen des arabischen Originals. Zu denken ist in diesem Kontext insbesondere an die „Rihla“ des prominenten Reformers, Übersetzers und Pädagogen Rifā‘a Rāfi' aț-Ṭahțāwī (1801-1873). Aṭ-Ṭahțāwī begleitete als Imam (religiöser Betreuer) zwischen 1826 und 1831 die erste ägyptische Studienmission nach Paris, welche der damalige ägyptische Herrscher Muhammad 'Alĩ im Rahmen seines ehrgeizigen Modernisierungsprogramms entsandt hatte. Seine Eindrücke von der Reise und dem Aufenthalt in Paris hat Aț-Ṭahțāwī in seinem 1849 erstmals erschienenen Bericht zusammengestellt, der einen kaum zu übersetzenden Titel in Reimprosa trägt Die Läuterung des Goldes in einer zusammenfassenden Darstellung von Paris, auf Arabisch Tahlīis al-ibrīz fì talhīiṣ Bārīz und deshalb meist kurz Rihla genannt wird. Nur in den ersten beiden Kapiteln gibt Aț-Ṭahțāwī Auskunft über das eigentliche Erlebnis der Reise und wie die ersten Hürden der Sprachaneignung überwunden wurden. Hauptsächlich beschreibt er aber die Sitten und Gebräuche der Pariserinnen und Pariser (Kleidung, Wohnung, Ernährung), bietet Informationen zum öffentlichen Leben und seiner Organisation, zum politischen System, zu Wohlfahrt oder Wissenschaften und Künsten. Er wird damit nicht nur zum Wissensvermittler (so hat er z.B. erstmals eine neue Terminologie für bis dahin in der arabischen Welt unbekannte Phänomene geschaffen bzw. vorgeschlagen), sondern auch zum Mittler zwischen den Kulturen. Nach seiner Rückkehr fungierte er als Leiter der von Muhammad 'Alī begründeten Sprachenschule und hat selbst, wie auch seine Schüler, einige französische Werke ins Arabische übertragen, darunter geographische und historiographische.

Im westlichen Kulturkreis ist die Erzählung Sindbad der Seefahrer am meisten bekannt, der die damals bekannten bzw. legendären Gewässer entdeckte und eroberte laut der Maxime „Wer wagt, gewinnt”. Diese Erzählung ist oft übersetzt und sogar mehrfach verfilmt worden und so zur Inspirationsquelle für viele Künstler geworden. Nur wenige können sich daran erinnern, dass die Erzählung als eigenständiges Werk entstanden war, dann aber vom französischen Übersetzter und Orientalisten Antoine Galland in seine Ausgabe der Erzählungen aus Tausendundeiner Nacht eingefügt wurde, die in den Jahren 1704-1717 unter dem Titel Les Mille et Une Nuit in Paris verlegt wurde. Erst von dem Zeitpunkt an etablierte es sich in der Literaturwelt 
als ein integraler Teil dieser Sammlung, denn die Übersetzung Gallands nutzten alle nachfolgenden europäischen Übersetzer. Die Erzählungen aus Tausendundeiner Nacht genossen große Popularität nicht nur in Frankreich, sondern in der ganzen westlichen Welt, woran die Tatsache der Niederlage der osmanischen Truppen bei Wien (1683) einen erheblichen Anteil hatte. Der Orient hörte auf, ein Symbol der Gefährdung zu sein, und die damit früher verbundenen Gefühle der Gefahr und Ängste der Europäer schlugen schnell in Faszination für ihre Exotik um und weckten das Interesse an der arabischen Welt.

R.B.: Das ist sicherlich richtig. Allerdings ist zu bedenken, dass diese Faszination für „den (arabischen/muslimischen) Orient” auch einer verzerrten, imaginären Vorstellung vom „Orient” Vorschub leistete, die - nach dem palästinensischen Literaturtheoretiker und kritiker Edward Said (vgl. sein Hauptwerk Orientalism von 1978) - partiell als Projektion westlicher Wunschphantasien zu verstehen ist. Diese und weitere stereotype Bilder, die bereits seit Jahrhunderten das Islam-Bild in der westlichen Hemisphäre geprägt hatten, dienten den europäischen Großmächten im imperialen Zeitalter schließlich einerseits der Selbstvergewisserung und andererseits dem Nachweis ihrer (vermeintlichen) zivilisatorischen Überlegenheit.

Zum Schluss möchte ich eine - einem breiteren Leserkreis unbekannte - Tatsache in-Erinnerung rufen, nämlich dass wir der arabischen erdkundlichen Literatur die erste Beschreibung der polnischen Gebiete in der Geschichte verdanken, und zwar in Gestalt eines Berichts von der Reise nach Mitteleuropa von Ibrāhīm, dem Mitglied der Gesandtschaft des Kalifen Al-Ḥakam II. zu Kaiser Otto I., die er in den Jahren 960-966 unternommen hat. In diesem Werk, das leider nicht vollständig erhalten ist, sondern nur fragmentarisch aus Überlieferungen späterer Autoren - Al-Bakrī, AlQazwīnī oder Al-Ḥimyārī - bekannt ist, finden wir eine Beschreibung Polens mit der Bezeichnung „Land des Mieszko” als „des weitläufigsten von slawischen Ländern”, das „reich an Nahrung, Fleisch, Honig und Feldern ist, und dessen Herrscher dreitausend Krieger hat, die in Verbände unterteilt sind, und ein Hundert von ihnen gleichen zehn hundert anderen Kriegern”. Und welches Bild des deutschen Gebietes liefert darin Ibrāhīm?

R.B.: Es gab bis dahin nur wenige Angaben zu den Gebieten, die heute zu Deutschland zählen. Die erste Erwähnung der „Franken”, worunter seit der Zeit Karls des Großen etwa alle westeuropäischen Gebiete nördlich von al-Andalus subsumiert wurden, gehen auf Adaptionen der Geographie des Ptolemäus (so z.B. bei dem oben erwähnten Al-Huwārizmī) zurück; etwas ausführlicher ist dann Al-Mas'ūdī. Gesandtschaften mit diesen Gebieten wurden anscheinend selten ausgetauscht; die diplomatischen Kontakte zwischen Karl dem Großen und Hārūn ar-Rašìd werden nur in fränkischen Quellen erwähnt. Ibrāhīm Ibn Ya'qūb aț-Ṭarțūšī, dem Namen nach ein jüdischer Bewohner Tortosas, verdanken wir tatsächlich die ersten detaillierten Beschreibungen west-, süd- und norddeutscher Städte, sowohl im Land der „Franken” (Ifranğ) als auch im Land der „Slawen” (Șaqāliba), zu denen damals die Araber auch norddeutsche Gebiete zählten. So beschreibt er etwa ausführlich die Abtei von Fulda und die Handelspraktiken in Augsburg. (Die dort übliche Auszeichnung von Waren erschien dem Autor z.B. eigentümlich.) Bekannt sind seine Beschreibungen über die Salzgewinnung in Soest. Zudem beschrieb er Mainz und die 
„slawischen” Städte Paderborn, Schleswig oder Magdeburg, wo er von Otto I. empfangen wurde. Über die Franken weiß er zu berichten, dass sie ein immens großes Reich beherrschten, das christlich geprägt sei, das Klima sei rau, aber die Ernte reichhaltig, zudem gebe es Silberminen, die Einwohner gehorchten dem König, der sich auf eine beachtliche, mutige Armee stütze. Allerdings moniert er die fehlende Hygiene - eine Bemerkung, die sich auch in späteren Berichten von Reisenden findet, was nicht wundert, wenn man bedenkt, dass Cordoba etwa zur gleichen Zeit bereits über eine große Anzahl öffentlicher Bäder verfügte. Eine Bemerkung zu den Frauen von Schleswig ist besonders hübsch. Der Autor schreibt, dass sich dort die Frau auf Eigeninitiative von ihrem Mann trennen könne, wann immer sie wolle.

Ich danke Ihnen, Frau Professor, für das Gespräch. 